(C) 2015 IEEE. Personal use of this material is permitted. Permission from IEEE must be obtained for all other uses, in any current or future media, including reprinting/republishing this material for advertising or promotional purposes, creating new collective works, for resale or redistribution to servers or lists, or reuse of any copyrighted component of this work in other works

http://dx.doi.org/10.1109/IISA.2015.7388023 


\title{
Diagnosis of Alzheimer's disease based on Virtual Environments
}

\author{
Juan Manuel Fernández Montenegro and Vasileios Argyriou \\ Faculty of Science, Engineering and Computing \\ Kingston University \\ Kingston upon Thames, UK \\ J.Fernandezmontenegro@kingston.ac.uk, Vasileios.Argyriou@kingston.ac.uk
}

\begin{abstract}
Alzheimer's screening tests are currently used by doctors as part of a thorough patient examination. These tests are performed periodically in specific time intervals trying to estimate the patient's condition and stage as early as possible. This work proposes a novel e-health Alzheimer's screening test based on virtual environments using new immersive technologies combined with advanced Human Computer Interaction (HCI) systems. These new tests are focused on the immersion of the patient in a virtual room, in order to mislead and deceive the patient's mind. As a result, two new tests are introduced demonstrating the wide range of screening mechanisms that could be designed using virtual environments. The proposed tests are focused on the evaluation of memory loss related to common objects, recent conversations and events, the diagnosis of problems in expressing and understanding language and the ability to differentiate between virtual worlds and reality. The proposed screening tests were evaluated and tested using both patients and healthy adults in a comparative study with state-ofthe-art Alzheimer's screening tests.
\end{abstract}

Keywords-Alzheimer's disease; Dementia; Early detection; Screening tests; HCI; Virtual environments

\section{INTRODUCTION}

Alzheimer is a dementia disease that usually affects elder people with the number of cases increasing significantly over the last decades. Dementia is a syndrome characterised by the decline of mental skills such as memory, reasoning, language or perceptual interpretation. Alzheimer is the most common type; accounts for an estimated 60 to 80 percent of cases, and one of the most noticeable symptoms is the difficulty learning new information. In addition, when the disease advances, there are other symptoms such as disorientation, mood and behaviour changes; confusion about events, date and place; suspicious about family, friends and caregivers; and difficulty speaking, writing and walking [1].

According to the World Health Organization, $1.43 \%$ of the estimated deaths around the world in 2030 will be caused by Alzheimer's disease or other dementias. The main characteristics of dementia are the decline of mental abilities [2] and people with dementia live five years on average [3]. Currently, when Alzheimer's disease (AD) is diagnosed, the neuronal damage is spread enough to make it irreversible [4]. Therefore, it is important to detect dementia at very early stages in order to reduce the deterioration speed.
In addition to the increasing mortality due to Alzheimer, the cost of dementia is another issue to take into consideration. The worldwide cost of dementia estimated in 2010 was US\$604 billion [5] and it was estimated to increase $85 \%$ by 2030 . These costs account for around $1 \%$ of the world's gross domestic product. Therefore, it is essential to develop affordable diagnosis and support tools limiting the increasing cost of dementia. The fact of being able to provide affordable and quality services, affects the whole health system. As a result, one of the proposed initiatives is focused on the implementation of e-health (the use of Information Communication Technology (ICT)) solutions reducing the cost trying to make the health systems universally accessible [6]. Amongst the e-health range of advantages, there are the improvement of the patients' lifestyle, the quality of care and efficiency (time saved) or the possibility of reducing the transfer time of medical data such as diagnostics.

In order to detect Alzheimer, different types of invasive and non-invasive tests are available. Invasive methods require obtaining data from the interior of the patient's body using injections, endoscopy, etc. These tests are not always safe and comfortable for the patient, while some of them are unbearably painful. On the other hand, non-invasive tests are harmless and despite the fact that some of them require the use of external devices during a certain period of time, these methods are more convenient during the diagnosis process [7]

The most common non-invasive Alzheimer tests for diagnosis are cognitive tests. These are question/task/problem based trying to measure patient's cognition. Cognition can be defined as the use of the information that has been previously collected by a person to make behavioural decisions [8]. Cognitive or Aptitude tests have high accuracy when it comes to Alzheimer's detection. Nevertheless, one of their weaknesses is the evaluation of cognitive reserve [4]. Cognitive reserve is the capacity of the brain to compensate brain damage. Another well know problem is the adaptability of the test according to the IQ of the patient since most of the tasks that integrate an Alzheimer's detection cognitive test usually are too simple to evaluate high IQ patients. The use of computerised tests helps to create intelligence adaptable cognitive tests [9]. Moreover, based on the available technology, it is possible to design new types and more effective tests using for example virtual environments (VEs). Virtual environments (VEs) provide additional advantages to 
cognitive tests, since it is possible to immerse the patient in a controlled situation, $[10,11,12]$.

In this paper, we propose novel cognitive and executive function based, non-invasive screening tests for early Alzheimer's diagnosis implemented as an e-health tool. These new tests are conducted in a virtual room due to the flexibility to create different tasks and the flow provided by VEs [10, 12]. As a result, an innovative and cost efficient e-health Alzheimer's detection application is introduced.

The remainder of this paper is organised as follows: section 2 describes previous related work on Alzheimer's diagnosis. Section 3 analyses the proposed methodology and in section 4 details on the evaluation process and the obtained results are presented. Section 5 gives some conclusion remarks.

\section{PREVIOUS WORK}

Early stage dementia detection has significant importance in order to reduce the progress speed [13]. The main types of tests can be separated to non-cognitive and cognitive tests. Cognitive tests encompass the methods that evaluate patients' cognition and whose procedures are non-invasive and easy to implement. Non-cognitive test is regarded any other method, invasive or non-invasive, used to detect and diagnose dementia.

Regarding the non-cognitive approaches, in $[1,14]$ some of the methods used to detect dementia are based on defining potential biomarkers that are acquired through invasive techniques. Other non-cognitive techniques presented in $[15$, 16] that provide accurate results are based on Magnetic Resonance Imaging (MRI). These techniques compare the head MRI data of the patient with the corresponding data of patients with Alzheimer using machine learning techniques. About the non-cognitive Alzheimer detection methodologies, monitoring approaches have been considered in the past. For example, as it was suggested in [13] sensors in patients' homes are used to monitor their behaviour and to identify certain events.

Despite the large range of dementia detection tests, the most common tests for early detection are the cognitive ones. These tests are based on problem solving tasks and questions, in order to detect a cognitive impairment. The Mini Mental State Examination (MMSE) is one of the most well-known and used tests [17]. The results provided by this test demonstrate high accuracy and specificity. Nevertheless, the results show a ceiling effect, since the test is not complex enough for high IQ patients [17]. Saint Louis University Mental Status (SLUMS) examination is another screening tool used to detect mild dementia similar to MMSE [18]. On the other hand, there are other cognitive tests focused mainly on a single cognitive task. For example, the memorisation of interacting objects in the Visual Association Test (VAT) [19] or the detection of the prevalence of the right ear when sounds are memorised by Alzheimer's patients during the Dichotic Listening test [20, 21].

Recently, due to the new advances on computer technologies, Virtual Environments (VEs) started to become part of medical tests and rehabilitation therapies. The work presented by I. Tarnanas, et al. in [11] demonstrates that the use of VEs is beneficial when it comes to early dementia detection. It is possible to improve the results from previous cognitive tests since it reduces the floor and the ceiling effects, creating tests that adapt to the patients' IQ; and it is possible to increase the immersion of the patient in the task that is in progress. Their work uses large screens to display the environment and some depth sensors to recognise the gestures of the patients' body. This approach requires the patient to move to the location where the facilities for this test are available, due to the fact that the components are not portable and cost efficient making it inappropriate for e-health applications

In our work, a novel non-invasive Alzheimer's detection screening test based on VEs is proposed. The Virtual Environment created for this test is a room that the patients can recognise and feel comfortable, such as the doctor office, a living room, etc. The use of new immersive technologies such as virtual reality glasses provides a huge improvement in immersing the subjects in a VE. The created tool is the first Alzheimer' detection screening test that provides a full immersion in Virtual Environments implying that the patients are able to be more focused on the tasks, while additional information related to the environment-patient interaction is obtained. Also, it is possible to adapt some of the tasks to the patient's IQ level during the test, avoiding the ceiling effect. The test is based on the examination of cognitive abilities. Therefore, the test is question and task based, trying to provide a user friendly and comfortable environment for the patients. Furthermore, the proposed tests do not consider only memory tasks, but introduce novel approaches based on the concept of differentiating between reality and a virtual world.

\section{METHODOLOGY}

Due to the fact that the new tests are based on virtual environment immersion, the technology chosen is related to games development. Thus, VR glasses are the devices used to increase the immersion, whereas a depth camera is utilised to track the patients' movements and animate their avatars (see figure 1). Since, Alzheimer's patients are mainly elder people; all tasks are performed seated on a chair requiring only minimal movements. Virtual reality glasses, depth sensor and a computer are the tools required to perform the tests. These tools are affordable and they can be reused by other patients, resulting in a low cost Alzheimer's detection application. The virtual reality glasses provide an extra pair of lenses for nearsighted users to avoid wearing their glasses with the virtual reality glasses.

The main scene was designed such as to recreate the doctor's office or any other real room that the patient can recognise (e.g. office, living room), as we can see in figure 2. Then, the tests were defined taking advantage of the virtual environment versatility, such as the absence of physical limits. Regarding the real environment, the patient is sitting on a comfortable chair about 2 meters in front of the depth sensor and is helped to place on the Virtual Reality glasses (see figures 3 and 4). Once the patient feels comfortable with the glasses, we proceed with the tests. The doctor (or test supervisor) has to be in front of the computer locally or remotely, in order to control the software application and has to take notes during the process typing details that may be useful later, since it is possible to obtain in that way extra information 
about the patient's behaviour. Despite most of the tests are automatic and the patient only has to follow the instructions, some actions, such as starting a new task or playing sounds, requires the interaction of the doctor/instructor in order to provide enough time to the patient to perform the actual tasks or to add a degree of randomness and adaptability to the patient.

All the proposed tests should be performed under the supervision of a healthy person following the instructions on the screen or the test's information sheets. Despite the fact that the test could be monitored by any person without mental impairments, it is recommendable that the supervisor during the tests to be a doctor either locality or remotely, since additional information may be obtained during the whole process. In this work, two novel tests are introduced: Virtual Objects Memorisation and Virtual vs Real Sounds.

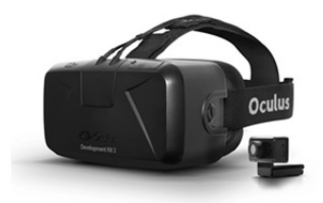

Oculus Rift DK2

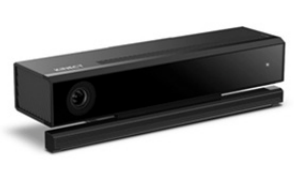

Kinect Sensor
Fig. 1. Virtual Reality glasses (Oculus Rift DK2) and Depth sensor (Microsoft Kinect 2 sensor) are the devices required to perform the tests.
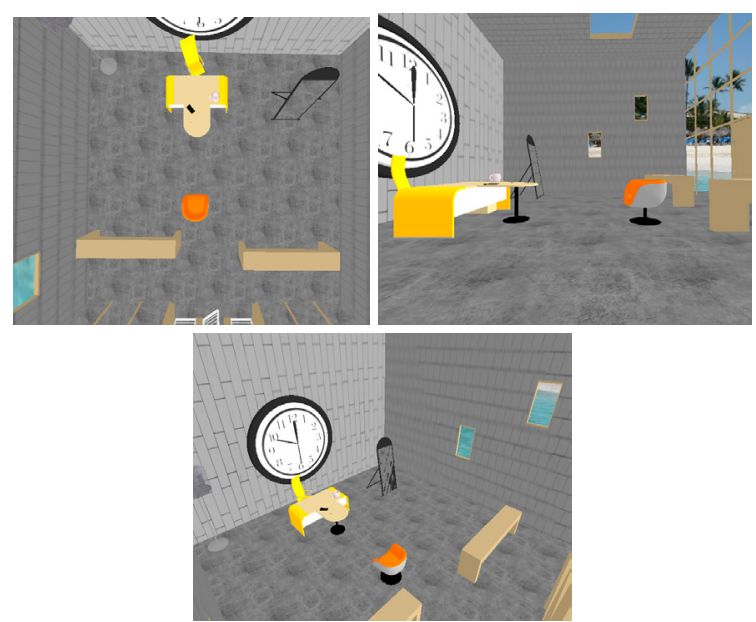

Fig. 2 Virtual Reality Room: Top down; Side; and Corner view.

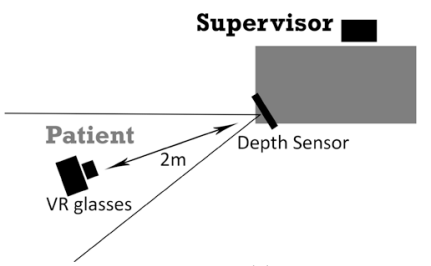

(a)

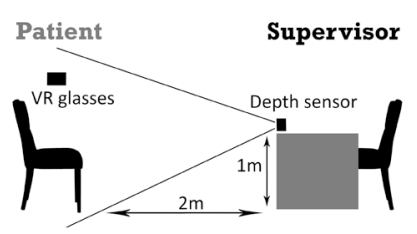

(b)

Fig. 3 (a) Top down view showing the position of the devices, and the location of the patient and the supervisor. Around the patient should be space to avoid collation with any real objects. (b) Side view showing the position of the devices, and the location of the patient and the supervisor. The depth sensor should be located at approximately 1 meter above the floor. The patient has to be approximately 2 meters away from the depth sensor in order to be in the view range.

\section{A. Virtual Objects Memorisation test (VOM)}

This test is formed by memory tasks and it is split into three steps. The first step of the memory test is the recognition of six different virtual objects. The objects are common used during daily activities making them easily identified and recognised. The objective of this first task is to check if the patient has problems in recognising quotidian objects (see figure 5). Once all the objects are recognised, a virtual monitor displays all of them in specific positions (the positions of these objects are the ones where they normally are located on a desk). Therefore, the patient is asked to memorise the objects but nothing is mentioned about their position.

During the second step, the subject is asked about the objects located at specific positions, in order to check the visual and the associative memory of the patient. In more details, the desk is shown in front of the patient and a number appears in the position, where an object was located and a list of the six possible objects is provided (see figure $5 \mathrm{c}$ ). The subjects are asked about the object that was located in the position indicated by the number and they have to select the name of the correct object. The fact that the objects were located in logical positions should help the user to select the right answers.

Finally, in the last task, interacting objects are placed in the virtual room and have to be located and memorised by the patient. Then, one of the objects is shown and the subject has to recall its interacting couple and their functionality. Once the third stage is completed, there is an extra task associated with the first step, where the patient is asked to recall the objects that were shown. The aim of the last two tasks is to evaluate the patient's short-term memory incorporating interacting objects and their functionalities. So, the novel part here is focused mainly on the combination of interacting objects with their functionalities.

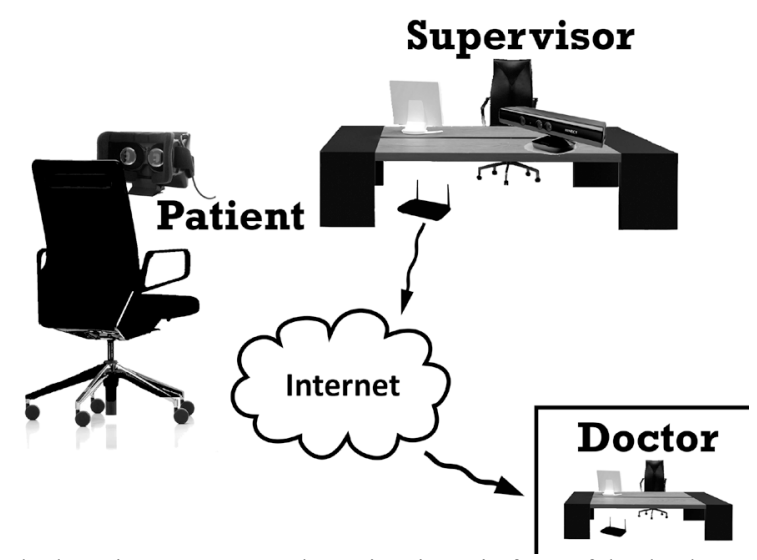

Fig. 4. Early detection Test setup. The patient is sat in front of the depth sensor, wearing the VR glasses. The supervisor (or the doctor) monitors the test from the computer or remotely as an e-health application.

Also, it should be mentioned that in order to proceed through the test, the subject has to select amongst different options. This selection is performed by aiming towards the desired option using the direction of view (scope), as we can see in figure 5 . Therefore, the patients have to move slightly the head to fulfil the tasks. 
This test provides in total 21 points, if all tasks are completed successfully. Each point is associated to the recognition of the objects, the recall of their position and the recall of the objects themselves and their functionality.

\section{B. Virtual vs Real Sounds test (VRS)}

The main objective of this test is to detect if the patient is able to discern between real and virtual sounds. Different sounds are played during the test, such as a clock ticking, the rain sound, etc (see table 1). The patient has to recognise and name all the sounds that they listen during the test. This part of the test tries to identify if the patient is still able to discern events through sounds. In addition, the virtual environment will provide some visual clues to help them with the identification (see figure 6). Once the subjects have identified all the sounds, they are asked about the origin of the sounds. During this stage, the source of some sounds is coming either from the virtual environment and the real world reproduced by the supervisor. This second part requires the patient to recall all the sounds that were played and to identify which ones were reproduced by the supervisor.

This test gives a maximum score of 6 points. One point is obtained for each object that is recognised and categorised properly as real or virtual.

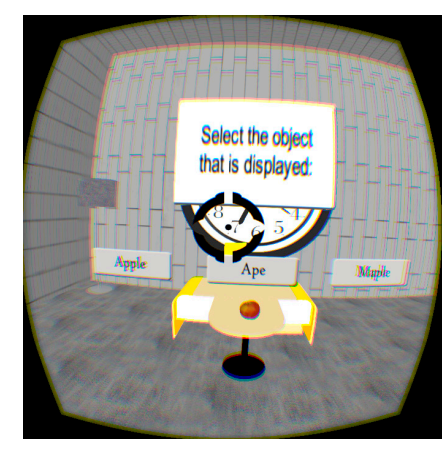

(a)

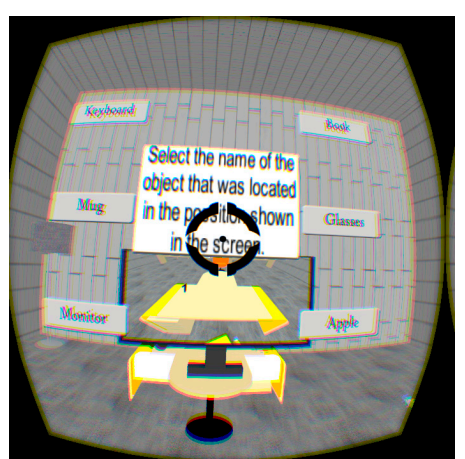

(c)

Fig. 5. (a) Virtual Object Memorisation tasks. Objects Recognition task where the patient has to identify and select the correct name of the object over the table. Virtual Object Memorisation tasks. (b) The objects in their respective location is shown in order the patient memorises the objects. (c) Objects location task where the patient has to select the name of the object that was shown on the screen
TABLE I. SOUNDS PLAYED DURING VRS TEST

\begin{tabular}{|l|l|}
\hline \multicolumn{1}{|c|}{ Sound } & \multicolumn{1}{c|}{ Origen } \\
\hline Ticking clock sound & Virtual \\
\hline Fly buzzing sound & Virtual \\
\hline Breaking glass sound & Virtual \\
\hline Closing door sound & Virtual \\
\hline Rain sound & Virtual \\
\hline Mobile phone & Real \\
\hline
\end{tabular}

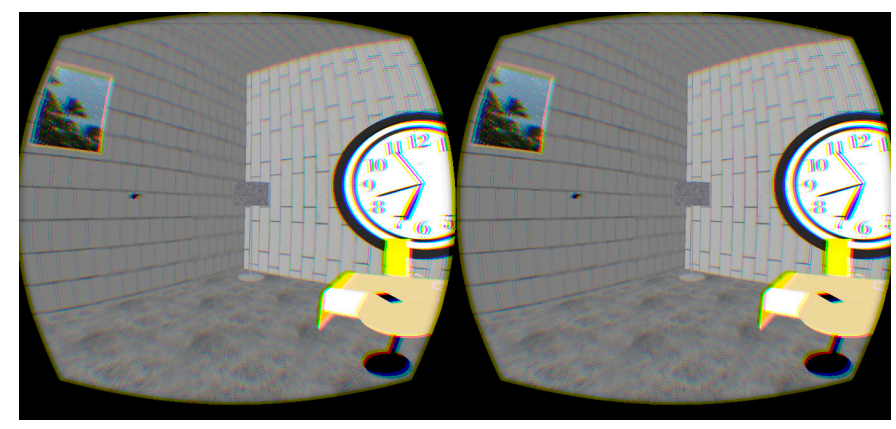

Fig. 6 Virtual vs Real Sounds test. The image shows 4 sound sources/clues of the 6 used (Clock, fly, rain and mobile phone). The mobile phone sound is reproduced by the test supervisor

\section{RESUlts}

\section{A. Participants}

The 20 participants of this test are aged between 23 and 82 years old including both healthy people and Alzheimer's patients diagnosed with mild dementia less than year ago. Furthermore, Alzheimer's patients in advanced conditions were tested but, the process was not possible to be completed. Half the subjects were male and the other half female of whom eleven had college or higher educational background and nine did not reach high school. It has to be mentioned that one of the patients was illiterate so the test was performed orally.

\section{B. Data}

The main data collected during the experiment are the individual scores for each test. As it was explained in section 3, each test has a maximum score and each task has associated to a different number of points. In more details, regarding the recognition and memory tasks each recalled and recognised object scores 1 point. The results have been normalised in order all the tests have results between 0 and 1 .

In addition, patients have been also tested using other wellknown state of the art Alzheimer screening tests in order to perform a comparative study and allow the evaluation of the proposed novel non-invasive diagnosis screening tests. All the tests were computerised so the patients have to use the computer through the whole process retaining also the same conditions among all tests. The screening tests used in our comparative study are Dr. Oz Memory Quiz [18, 22]; Visual Association Test [19]; and Dichotic Listening Test (DLT) [20].

\section{Results}

The mean results for each test in relation to the subject's health status are shown in Table 2. It is observed that healthy patients have achieved results close to the maximum score of 
each test and the Alzheimer's patients have obtained significantly lower scores in comparison with the health participants. For instance, healthy subjects have obtained a mean of .92482 whereas the Alzheimer's subject has scored .23810 in the Virtual Objects Memorisation test. This separation between results is also apparent on the other state of the art tests, such as the relation .98684-.37500 (Healthy Alzheimer) on the Dichotic Listening test.

TABLE II. MEAN OF THE TESTS REGARDING THE HEALTHY AND ALZHEIMER PATIENTS

\begin{tabular}{|c|c|c|c|c|c|c|}
\hline TYPE & Age & DrOz & VAT & DLT & VOM & VRS \\
\hline Healthy & 42.47 & .90095 & .89473 & .98684 & .92482 & .83331 \\
\hline Alzheimer & 78.00 & .52940 & .00000 & .37500 & .23810 & .33330 \\
\hline
\end{tabular}

Table 3 demonstrates that the $p$-value of the novel tests rejects the Null Hypothesis. Therefore, it proves that the novel tests' results are correlated with the healthy and Alzheimer status of the patients.

TABLE III. T-TEST FOR EACH TEST ACCORDING TO THE HEALTHY AND ALZHEIMER'S CASES. THE P-VALUE (SIG. (2-TAILED)) IS LESS THAN 0.01 SO THE NULL HYPOTHESIS IS REJECTED

\begin{tabular}{|c|c|c|c|c|c|}
\hline & \multicolumn{5}{|c|}{ t-test for Equality of Means } \\
\hline & $t$ & $f$ & Sig. (2-tailed) & Mean Difference & Std. Error Difference \\
\hline $\begin{array}{r}\text { DrOz } \text { e.v. } a^{a} \\
\text { e.v.n. } a^{b}\end{array}$ & 4.347 & 18 & .000 & $\begin{array}{l}.3715526 \\
.3715526\end{array}$ & .0854726 \\
\hline $\begin{array}{rc}\text { VAT } & \text { e.v.a } \\
& \text { e.v.n.a }\end{array}$ & 4.164 & 18 & .001 & $\begin{array}{l}.8947316 \\
.8947316 \\
\end{array}$ & .2148737 \\
\hline $\begin{array}{rc}\text { DLT } & \text { e.v.a } \\
& \text { e.v.n.a }\end{array}$ & 21.333 & 18 & .000 & $\begin{array}{l}.6118421 \\
.6118421\end{array}$ & .0780741 \\
\hline $\begin{array}{r}\text { VOM } \begin{array}{r}\text { e.v.a } \\
\text { e.v.n.a }\end{array} \\
\end{array}$ & 7.418 & 18 & .000 & $\begin{array}{l}6867211 \\
6867211 \\
\end{array}$ & .0925755 \\
\hline $\begin{array}{cc}\text { VRS } & \text { e.v.a } \\
& \text { e.v.n.a }\end{array}$ & 4.386 & 18 & .000 & $\begin{array}{l}.5000105 \\
.5000105\end{array}$ & .1139947 \\
\hline
\end{tabular}

b Equal Variances Not Assumed

On the other hand, left part of Table 4 shows that the mean of the scores of the tests for subjects aged above 50 years old are always lower than the scores obtained by patients under 50 years old. However, right part of Table 4 demonstrates that the correlation of the results and the age of the patients is not clear, since the p-values do not reject the Null hypothesis.

The correlation between the DrOz, VAT and DLT tests, and the novel ones is presented in Table 5, where the Pearson correlation has been calculated, including the correlation coefficient and the $p$-value. All the novel tests result high correlation value with the existent tests and the p-value supports this correlation.
TABLE IV. MEAN AND STANDARD DEVIATION ACCORDING WITH 2 AGE GROUPS (OVER AND UNDER 50 YEARS OLD); AND P-VALUE IN RELATION WITH AGE GROUPS

\begin{tabular}{|c|c|c|c|c|c|c|}
\hline & Age & $\mathbf{N}$ & Mean & $\begin{array}{l}\text { Std. } \\
\text { Deviation }\end{array}$ & & Sig. (2-tailed) \\
\hline \multirow[t]{2}{*}{ DrOz } & $>=50$ & 8 & .816200 & .1554446 & e.v. $a^{a}$ & .033 \\
\hline & $<50$ & 12 & .926492 & .0509407 & e.v.n. $a^{b}$ & .088 \\
\hline \multirow[t]{2}{*}{ VAT } & $>=50$ & 8 & .770825 & .3876870 & e.v.a & .325 \\
\hline & $<50$ & 12 & .902775 & .1940844 & e.v.n.a & .395 \\
\hline \multirow[t]{2}{*}{ DLT } & $>=50$ & 8 & .906250 & .2167916 & e.v.a & .198 \\
\hline & $<50$ & 12 & .989583 & .0258944 & e.v.n.a & .314 \\
\hline \multirow[t]{2}{*}{ VOM } & $>=50$ & 8 & .797638 & .2516588 & e.v.a & .052 \\
\hline & $<50$ & 12 & .952383 & .0574379 & e.v.n.a & .128 \\
\hline \multirow[t]{2}{*}{ VRS } & $>=50$ & 8 & .729138 & .1979503 & e.v.a & .061 \\
\hline & $<50$ & 12 & 861092 & .0962259 & e.v.n.a & .113 \\
\hline
\end{tabular}

b Equal Variances Not Assumed

\section{Qualitative evaluation of the proposed system}

At the end of the test, patients were asked to evaluate the application. They had to fill a form evaluating the quality of the application in terms of interaction; the simplicity of the instructions/process and the comfort of the glasses. Additionally, the patients had the option to write any additional comments about the application.

The average evaluation of the application was positive. Some of the subjects commented that the glasses were not comfortable at the end of the test and that the graphics could be improved. Nevertheless, most of the participants evaluated the application as motivating and interesting; and the interaction and instructions as simple and easy to understand.

TABLE V. STATE OF THE ART AND NOVEL TESTS CORRELATION.

\begin{tabular}{|c|c|c|c|c|c|}
\hline & DrOz & VAT & DLT & VOM & VRS \\
\hline DrOzCorrelation Coefficient & 1.000 & $.467 *$ & $.677 * *$ & $.589 * *$ & $486^{*}$ \\
\hline Sig. (2-tailed) & & .038 & .001 & .006 & .030 \\
\hline$N$ & 20 & 20 & 20 & 20 & 20 \\
\hline VAT Correlation Coefficient & $467 *$ & 1.000 & $.726 * *$ & $.666^{* *}$ & $602 * *$ \\
\hline Sig. (2-tailed) & .038 & & .000 & .001 & .005 \\
\hline$N$ & 20 & 20 & 20 & 20 & 20 \\
\hline DLT Correlation Coefficient & $677 * *$ & $.726 * *$ & 1.000 & $832 * *$ & $688^{* *}$ \\
\hline Sig. (2-tailed) & .001 & .000 & . & .000 & .001 \\
\hline$N$ & 20 & 20 & 20 & 20 & 20 \\
\hline VOMCorrelation Coefficient & $.589 * *$ & $.666 * *$ & $.832 * *$ & 1.000 & $.806^{* *}$ \\
\hline Sig. (2-tailed) & .006 & .001 & .000 & . & .000 \\
\hline$N$ & 20 & 20 & 20 & 20 & 20 \\
\hline VRS Correlation Coefficient & $486^{*}$ & $.602 * *$ & $688 * *$ & $.806^{* *}$ & 1.000 \\
\hline Sig. (2-tailed) & .030 & .005 & .001 & .000 & . \\
\hline$N$ & 20 & 20 & 20 & 20 & 20 \\
\hline
\end{tabular}

Table 6 presents the overall evaluation of the tool performed by the participants. The interaction with the application (keyboard, mouse, depth sensor and virtual reality glasses), the quality of the instructions (written and/or oral provided by the supervisor) and the comfort of the VR glasses was evaluation from 0 points (really bad) to 5 points (really good). The evaluation obtained is positive, since the average 
result of all the categories is above 3 points, with elder people being more enthusiastic with new technologies probably due to the completely new to them features and capabilities.

TABLE VI. TOOL EVALUATION BY THE PARTICIPANTS. FOUR CHARACTERISTICS WERE EVALUATED FROM 0 (VERY BAD) TO 5 (VERY GOOD)

\begin{tabular}{|c|c|c|c|c|}
\hline TYPE & Mouse Inter & Kinect Inter & Instructions & VR Glasses \\
\hline$>=\mathbf{5 0}$ & 4.17 & 4.00 & 4.43 & 3.86 \\
\hline$<\mathbf{5 0}$ & 4.33 & 3.33 & 4.17 & 3.50 \\
\hline
\end{tabular}

\section{CONCLUSIONS}

Virtual Environments (VEs) start to be part of medical treatments or diagnosis methods, and they have been proved to be considerably positive to apply techniques in secure and controlled environments; helping to increase the effectivity of the related tests. These facts have been further proved with our application, since the results obtained show that it is possible to create an accurate e-health screening Alzheimer's diagnostic system. In this work novel methods for detection of Alzheimer's disease based on Virtual Environments were introduced. The flexibility of VEs has demonstrated to solve the ceiling effect present in most used cognitive test. The proposed tests are focused on the evaluation of memory loss related to common objects, recent events, the diagnosis of problems in expressing and understanding language and the ability to differentiate between virtual worlds and reality. The proposed approaches were evaluated in a comparative study with well-known state of the art cognitive tests. Finally, a novel e-health tool has been introduced and developed that proves the benefits of e-health services such as the improvement of patient's daily life, avoiding painful tests; its affordable cost, since the difference price of the testing devices in comparison with other projects using HCI systems is considerable, moreover, the plausible decrease of the VR glasses due to the foreseen peak next years; and the wide accessibility that allows the patient to be tested at home.

\section{REFERENCES}

[1] Alzheimer's Association (2014). Alzheimer's \& Dementia. Available from: <http://www.alz.org/>. [25 November 2014]

[2] Whalley L.J. \& Breitner J. C. S. (2009). Fast Facts: Dementia. Health Press.

[3] Alzheimer's Research UK (2012). Alzheimer's and dementia: your questions answered. Alzheimer's Research UK

[4] Tarnanas I., Tsolaki M., Nef T., M Müri R. \& Mosimann U. P. (2014) "Can a novel computerized cognitive screening test provide additional information for early detection of Alzheimer's disease?" Alzheimer's \& Dementia.

[5] Wimo A., \& Prince M. J. (2010). World Alzheimer Report 2010: the global economic impact of dementia. Alzheimer's Disease International.
[6] Lewis T., Synowiec C., Lagomarsino G. \& Schweitzer J. (2012). Ehealth in low-and middle-income countries: findings from the Center for Health Market Innovations. Bulletin of the World Health Organization, 90(5), 332-340.

[7] López-de-Ipiña K., Alonso J. B., Barroso N., Faundez-Zanuy M., Ecay M., Solé-Casals J., Travieso C. M., Estanga A. \& Ezeiza A. (2012). New approaches for Alzheimer's disease diagnosis based on automatic spontaneous speech analysis and emotional temperature. In Ambient Assisted Living and Home Care (pp. 407-414). Springer Berlin Heidelberg.

[8] Rowe C. \& Healy S. D. (2014). Measuring variation in cognition. Behavioral Ecology, 25(6), 1287-1292.

[9] Wild K., Howieson D., Webbe F., Seelye A. \& Kaye J. (2008). Status of computerized cognitive testing in aging: a systematic review. Alzheimer's \& Dementia, 4(6), 428-437.

[10] Taekman J. M. \& Shelley K. (2010). Virtual environments in healthcare: immersion, disruption, and flow. International anesthesiology clinics, 48(3), pp: 101-121.

[11] Tarnanas I., Schlee W., Tsolaki M., Müri R., Mosimann U., \& Nef T. (2013). Ecological validity of virtual reality daily living activities screening for early dementia: longitudinal study. JMIR Serious Games, $1(1)$, e1.

[12] Weibel D. \& Wissmath B., "Immersion in Computer Games: The Role of Spatial Presence and Flow," International Journal of Computer Games Technology, vol. 2011, Article ID 282345, 14 pages, 2011. doi:10.1155/2011/282345

[13] Abe Y., Toya M. \& Inoue M. (2013). Early detection system of senile dementia by behavior sensing. Consumer Electronics (ISCE), 2013 IEEE 17th International Symposium. pp: 67-68

[14] Han S. D., Gruhl J., Beckett L., Dodge H. H., Stricker N. H., Farias, S. \& Mungas D. (2012). Beta amyloid, tau, neuroimaging, and cognition: sequence modeling of biomarkers for Alzheimer's Disease. Brain imaging and behavior,6(4), 610-620.

[15] Unay D. \& Ekin A. (2011).Dementia diagnosis using similar and dissimilar retrieval items. In Biomedical Imaging: From Nano to Macro. pp: 1889-1892

[16] Akgul C. B. \& Ekin A. (2010). A Probabilistic Information Fusion Approach to MR-based Automated Diagnosis of Dementia. In Pattern Recognition (ICPR).pp: 265-268

[17] Fountoulakis K. N., Tsolaki M., Chantzi H. \& Kazis, A. (2000). Mini mental state examination (MMSE): a validation study in Greece. American Journal of Alzheimer's Disease and Other Dementias, 15(6), pp: 342-345.

[18] Cruz-Oliver D. M., Malmstrom T. K., Roegner M., Tumosa N. \& Grossberg G. T. (2014). Cognitive deficit reversal as shown by changes in the Veterans Affairs Saint Louis University Mental Status (SLUMS) examination scores 7.5 years later. Journal of the American Medical Directors Association, 15(9), 687-e5.

[19] Lindeboom J., Schmand B., Tulner L., Walstra G., \& Jonker C. (2002) Visual association test to detect early dementia of the Alzheimer type. Journal of Neurology, Neurosurgery \&Psychiatry 73(2) pp: 126-133

[20] Duchek J. M. \& Balota D. A. (2005). Failure to control prepotent pathways in early stage dementia of the Alzheimer's type: evidence from dichotic listening. Neuropsychology, 19(5), pp: 687.

[21] Oppy G. \& Dowe D. (2011). The turing test. The Stanford Encyclopedia of Philosophy.

[22] Dr Oz (2014). Dr Oz Memory Quiz. Available at: www.doctoroz.com. 